\title{
El papel de los centros de poder en el proceso de toma de decisión de las políticas públicas y en la creación de la agenda política
}

\author{
Adela Romero Tarín \\ UNIVERSIDAD DE ALICANTE, ALICANTE, ESPAÑA \\ adela.romero@ua.es \\ José Manuel Canales Aliende \\ UNIVERSIDAD DE ALICANTE, ALICANTE, ESPAÑA \\ jm.canales@ua.es
}

Resumen: El proceso de toma de decisión por parte de los gobiernos marca el rumbo de las políticas públicas. En los centros de poder se priorizan qué políticas públicas se van a implementar a través de una selección de instrumentos, actores y recursos. Los centros de poder están compuestos por decisores políticos, y representantes de las diferentes esferas sociales y económicas, siendo el espacio de reflexión donde las problemáticas sociales comienzan a orientarse hacia su resolución. Se decide en estos espacios qué demandas sociales deben ser solventadas a corto, medio o largo plazo, y por tanto, si estas deben incluirse en la agenda política y su prioridad. Este artículo indaga sobre la importancia de estos centros de poder que protagonizan la génesis de las políticas públicas, cuestionando cómo influyen en la elaboración final de la agenda política a partir de los factores de liderazgo y tecnocracia. En este texto se analiza el papel que los centros de poder desarrollan en el proceso de toma de decisión, siendo entendidos como el timón del gobierno, aquellos capaces de impulsar los objetivos prioritarios de manera coherente. Estos pueden ser un valioso instrumento para asegurar que las políticas del gobierno sean efectivamente implementadas, y produzcan resultados exitosos para los ciudadanos (Alessandro et al., 2014:16). Esta propuesta investigadora emplea una metodología cualitativa a partir de un enfoque descriptivo, analítico y crítico, estructurado en cinco epígrafes, incluyendo referencias actuales del campo de las políticas públicas y de las ciencias políticas.

Palabras clave: políticas públicas, toma de decisiones, centros de poder, tecnocracia, liderazgo, grupos de presión.

Abstract: The decision-making process by governments marks the direction of political actions. In the centers of power, priority is given to which public policies will be implemented through a selection of instruments, actors and resources. The centers of power are composed of political decision makers, and representatives of the different social and economic spheres. These power centers are the space for reflection where social problems begin to be oriented towards their resolution. It is decided in these spaces that social demands must be resolved in the short, medium or long term, and therefore if they should be included in the political agenda. This article inquires about the importance of these centers of power that star in the genesis of public policies, also questioning how they influence the final elaboration of the political agenda based on the following factors: leadership, technocracy, historical context and pressure 
groups. This paper analyzes the role that power centers develop in the decision-making process, being understood as the helm of the government, those capable of driving the priority objectives in a coherent manner. These can be a valuable instrument to ensure that government policies are effectively implemented, and produce successful results for citizens (Alessandro et al., 2014:16). This research proposal employs a qualitative methodology based on a descriptive, analytical and critical approach, structured in five sections, including references in the field of public policy and political science.

Keywords: public policies, decision making, power centers, technocracy, leadership, lobbyists.

\section{Introducción}

l proceso de toma de decisión de una política pública requiere una reflexión,

una planificación y una aplicación responsable. Una política sin presupuesto, información, propaganda, sensibilización, rendición de cuentas, y que además, no esté regulada por normas legales, no puede definirse como una política pública. Esto podrá ser entendido como cualquier otra actividad, menos como una política. Por esta razón, su proceso de decisión, es el inicio para la resolución de problemas gubernamentales al conocer la implicación de instrumentos para su regulación económica y fiscal (Romero Tarín y Fontaine, 2017), actores institucionales y no institucionales (Peters, 2005), marcos jurídicos, agendas ciudadanas y políticas (Kingdon, 1984) e incluso factores contextuales, históricos y procedimental de los estados (Zittoun, 2009); son elementos que se combinan en este proceso para tomar las decisiones más adecuadas por los gobiernos en la implementación y ejecución de las políticas públicas.

Este artículo emplea una metodología cualitativa, a partir de un enfoque descriptivo, analítico y crítico, sin renunciar a un carácter divulgativo, estructurado en cinco epígrafes, incluyendo referencias y fuentes actuales del campo de las políticas públicas y de las ciencias políticas para proponer una serie de reflexiones sobre los centros de poder y su relación con las tomas de decisión, y la creación de la agenda política, descrita esta vinculación como un período cortoplacista, sin profundización en la adecuada división de responsabilidad y en la toma de decisión en el ámbito público. Además, también profundiza en la idea de que las políticas públicas son una manifestación y acción pública que forman parte de los estados, de los sistemas político-administrativos, y por tanto no son autónomas sino más bien, una parte del todo.

Cuando nos planteamos el desarrollo de este artículo, partimos del debate de principios del siglo XX entre democracia y tecnocracia, y también el producido a mediados del mismo siglo, entre política y administración pública, y entre élites políticas y élites administrativas en el contexto de la crisis de las democracias clásicas. 
Ahora bien, esas viejas discusiones reaparece y se le adhieren nuevas amenazas, como la de los grupos de presión o lobbies que desean manipular y aprovechar los recursos de los estados para satisfacer intereses privados; también la aparición de las fake news ha cambiado la mirada general, donde la verdad y la confianza en la comunicación entre ciudadanía-política se banaliza, y la reactividad de ideologías ya superadas en la estados democráticos resultado de una carencia de legitimidad hacia la política y a los estados, como son los neofascismos, neototalitarismos, neocomunismos, y populismos de distinto signo; junto al terrorismo internacional o yihadismo (entonces fue el anarquismo y el bolchevismo), resultando de todo ello, la llamada «crisis de la democracia representativa».

Este escenario descrito acontece en un nuevo contexto y marco de visión de lo público. En primer lugar, cabe señalar ante todo, que son hechos estructurales, fundamentales y novedosos aunque no los únicos como variables explicativas, siendo los más destacados los siguientes: a) la sociedad se ha transformado en su estructura y funcionamiento caracterizándose por las notas básicas siguientes: complejidad; diversidad, especialmente la multiculturalidad; pluralidad; el dinamismo del cambio social; la incertidumbre social y la ruptura del contrato social existente desde la Segunda Guerra Mundial; el carácter líquido o difuso de la sociedad (Bauman, 2004); y la multipolaridad; b) la globalización y sus múltiples efectos, afectando en particular a la soberanía y a la capacidad decisoria de los Estados y de las naciones sin una sociedad civil planetaria bien estructurada y potente (Peters y Pierre, 2002); c) la digitalización y sus retos; las redes sociales e internet han transformado notablemente todos los procesos sociales, políticos y económicos (Peters, 2005); d) la integración mundial de los mercados y la interdependencia de las sociedades predominantemente urbanas, y sobretodo tendentes a las megalópolis; e) y a su vez, se han producido dos cambios paradigmáticos o de visión de lo público que deben tenerse en cuenta dada su relevancia, a saber: el paradigma de la gobernanza (Canales Aliende, 2015) en el que se incardina toda la acción pública, y en la que aparece emergente y con fuerza el llamado Tercer sector, o en el que la ciudadanía y la sociedad civil participarán en lo público. La gobernanza implica «el gobierno relacional» o relacionado con el Tercer sector. Hoy pues, fruto de lo anterior, se han incorporado nuevos actores, nuevos movimientos sociales y nuevas instituciones que deciden y participan en lo público y en lo estatal (Gómez Calvo y Romero Tarín, 2016). El segundo cambio paradigmático, describe que lo público ha dejado de considerarse como equivalente y limitado al Estado y a su aparato administrativo. Hoy, un espacio público no estatal puede representarlo el Tercer sector, dejando atrás esa dicotomía clásica, sólo es Estado o es mercado. Por otro lado, éste es un concepto más amplio que el de empresa de carácter lucrativo, y no puede confundirse tampoco con el de sociedad de mercado. 


\subsection{El análisis del proceso de adopción de decisiones en el ámbito público y en las políticas públicas}

Los centros de poder realizan la función de decidir y priorizar las acciones a implementar por la administración pública, a través de los procesos de adopción de decisión que los políticos resuelven. Para realizar dicha tarea cabe formularse las siguientes preguntas básicas con carácter previo a una decisión definitiva, para luego diseñarla: a) quién o quiénes toman las decisiones; b) qué legitimación tienen los decisores, y si es propia o delegada; c) cuándo se decide y el cronograma de la decisión prevista en su caso; d) dónde se decide; e) cómo deciden; f) quiénes y cómo asesoran, en su caso a los decisores; g) existe o no información, debate y participación pública del tema a decidir, o no; $y$ h) qué documentación o información, estadística e indicadores analizan los decisores.

Una vez clarificadas estas preguntas o cuestiones iniciales, comenzaría propiamente el proceso de adopción de decisiones el cual a su vez podría tener entre otras las siguientes características: a) muy reglado o no; b) formal o informal; y c) más transparentes y abiertos, o no.

Además, hay que tener en cuenta que intervienen muchos actores en el proceso de decisión y creación de agenda, por esta razón, su complejidad es mayor. Los decisores no serán los ejecutores de la decisión tomada, y para facilitar su implementación y ejecución por los actores e instituciones implicados, estos deben tener una información concreta, precisa, clara, y entendible para responder a los objetivos planteados en la fase de formulación (Lindblom, 1994), para evitar así, equívocos en su interpretación y comprensión, lo cual pueda producir disfunciones, errores e incumplimientos en su aplicación. La flexibilidad es posible y necesaria en la gestión pública, siempre dentro del marco de la estrategia decidida y señalada previamente, no puede ser confundida con la improvisación, la oportunidad irresponsable y/o el deseo caprichoso.

\section{2. La etapa previa a la adopción de decisiones}

En esta fase de adopción de decisiones pueden darse diferentes situaciones, dependiendo de factores como: la temporalidad, siendo más duradera en el tiempo o no; el procedimiento, regulado o no; su aspecto racional/legal, formal o no; y por su composición, cuando intervienen o pueden intervenir no sólo los técnicos y los expertos, sino también la ciudadanía a través de distintos medios y causas, como expresión de la democracia participativa. El papel de los directivos y gerentes públicos, de los asesores y de los expertos en la fase previa a la adopción de decisiones, replantea, si bien en un nuevo contexto, el viejo dilema de la relación entre política y Administra- 
ción, o entre políticos y funcionarios (Baena del Alcázar, 2004; Martínez Puon, 2011; Ramió, 2012). El rol ideal es que los políticos tomen decisiones y los funcionarios les asesoren honestamente, pero esta idea de separación de tareas y de colaboración leal no es así en la práctica, ya que hay influencias y presiones mutuas.

Existe mucha documentación e información, la dificultada es cómo se selecciona, y si es rigurosa, y no interesada (Méndez Juez, 2016). En este sentido Vaillancourt (2011) en palabras de Pont Vidal (2017) incluye que un elemento de innovación en este proceso sería la coproducción de políticas públicas, a través de la participación de diversos actores de la sociedad civil en su diseño y también en las decisiones de la gestión de las políticas públicas (Pont Vidal, 2017:75), por otro lado, a esta propuesta de la coproducción, Ostrom (2011) la describe como un proceso que genera un valor público y establece un nexo entre las necesidades, los recursos, y las motivaciones de los ciudadanos junto a la propia gestión u organización de las políticas públicas.

No obstante, los directivos y los expertos asesoran a los gobiernos a través de diversos instrumentos: indicadores, informes, documentos, experiencias exitosas conocidas, etc. pudiéndose hacer a través de grupos de trabajo, comités, comisiones, etc. ya sea de carácter pluridisciplinar o no.

Es de gran utilidad además, que estas propuestas y sugerencias se plasmen en un plan estratégico con objetivos claramente identificados, y junto a una financiación aprobada por los gobiernos acordes con las agendas gubernamentales. Evidentemente, la existencia de empleados públicos meritocráticos y profesionales coadyuvará sin duda a una toma de decisiones rigurosa y objetiva, basada en las opiniones y alternativas posibles en función de la información y la documentación existente, seleccionada y utilizada por éstos. Además, será más eficaz en el ámbito de instituciones sólidas y consolidadas. Por todo ello, es muy útil que existan previamente unos protocolos de actuación y de ética profesional (Pasquino, 2000), y que estos órganos colegiados técnicos de carácter administrativo actúen conforme a un plan estratégico con objetivos claros aprobados previamente por los órganos y autoridades políticas.

Abundando en lo anterior, en el ámbito concreto de las políticas públicas, es de interés mencionar lo señalado por la OCDE (Organización para la Cooperación y el Desarrollo Económicos) respecto a la participación ciudadana en el proceso público decisional: «las relaciones entre las instituciones públicas y los ciudadanos constituyen para los gobiernos, una prioridad importante. En los últimos años, los ciudadanos y las organizaciones de la sociedad civil tienen cada vez mayor presencia, plantean problemas, formulan reivindicaciones y se esfuerzan por incidir en las decisiones de los operadores. Simultáneamente, los ciudadanos participan cada vez menos en los procesos democráticos formales. La tasa de participación electoral, por ejemplo, va disminuyendo. Ante esta pérdida de confianza, los gobiernos se ven 
obligados a establecer nuevas relaciones con los ciudadanos. Se dan cuenta también que la participación ciudadana puede ofrecer inmensas posibilidades para la toma de decisiones, sobre todo en un mundo cada vez mas complejo...» (OCDE, 2001:10). La participación ciudadana ofrece diversas modalidades e instrumentos, siendo en el ámbito local una herramienta digna de consideración, a través de los llamados presupuestos participativos, los cuales a pesar de sus limitaciones e insuficiencias en ocasiones, han sido una vía muy válida y eficaz de participación y opinión ciudadana.

No obstante, el presupuesto participativo fue un «experimento que empezó en los ochenta en América Latina, y que se ha extendido por todo el mundo, incluso a ciudades de América del Norte y Europa. Y, aunque estas prácticas han obrado a una escala muy pequeña y han afectado a una fracción minúscula de la financiación total de la ciudad, ejemplifican un modelo de buenas prácticas de gobernabilidad, y ofrece un camino hacia una mayor participación cívica (...)» (Curtis, 2018:11).

Entre las múltiples modalidades posibles y básicas de participación ciudadana, la OCDE, ha señalado las siguientes: «a) la información; b) la consulta; y c) la participación activa» (OCDE, 2001:17-18). Esta última: «(..) se refiere a que los mismos ciudadanos jueguen un rol en la elaboración de políticas públicas: por ejemplo, proponiendo alternativas. Sin embargo, tanto la definición de una política como la responsabilidad final sigue siendo responsabilidad del gobierno. La participación ciudadana en el proceso de toma de decisiones crea una relación bidireccional elaborada por las instituciones públicas y los ciudadanos, que se fundamenta en el principio de la coparticipación» (OCDE, 2001:18).

Así pues, mediante una participación real y no simbólica, la ciudadanía se siente copartícipe, es decir copropietaria de su destino, y stateholders del funcionamiento de las instituciones públicas, lo cual es además una manifestación relevante de la democracia participativa o de calidad; evitando por ende, los peligros de una posible desconfianza ciudadana que puede corroer los cimientos de la democracia, haciéndoles partícipes en los centro de poder en las tomas de decisiones.

Ahora bien, la participación activa se refiere a que los mismos ciudadanos jueguen un rol en la elaboración de políticas públicas, por ejemplo proponiendo alternativas. Sin embargo, tanto la definición de una política como la decisión final sigue siendo responsabilidad del gobierno. La participación ciudadana en el proceso de toma de decisiones crea una relación bidireccional elaborada por las instituciones públicas y los ciudadanos y se fundamenta en el principio de la coparticipación (OCDE, 2001).

Por otro lado, y añadido a lo precedentemente expuesto, coadyuvará sin duda notablemente al proceso de toma de decisiones objetiva y rigurosa por la clase política, la existencia de una clase gerencial, junto a una directiva profesional, meritocrá- 
tica, con valores y cultura de lo público, siendo un aspecto relevante actual del mismo su capacidad y habilidad para el liderazgo político (Méndez, 2013; González, 2013; Canales Aliende y Romero Tarín, 2014).

\subsection{Algunos factores destacados en el proceso de adopción de decisiones en los centros de poder}

En todo sistema democrático real y no meramente simbólico, le corresponde a la clase política en virtud de su legitimidad adquirida en las urnas, ya sea de forma directa o indirecta por el voto de la ciudadanía, la adopción de decisiones y por ende, de su responsabilidad ex post. El político atiende las diversas circunstancias, con un mayor o menor grado de discrecionalidad (a no confundir con la arbitrariedad y la prevaricación, a veces encubiertas) para poder decidir y optar entre varias opciones posibles. Si bien esto antes señalado, es lo normal y general, a veces no existe un margen posible de discrecionalidad, y ello por razones técnicas o excepcionales (así los supuestos entre otros de: fuerza mayor, máxima urgencia, tragedia sobrevenida, seguridad nacional, epidemia, etc.) que le obligan a decidir y actuar en una sola dirección y vía única posible.

Actualmente, y como resultado de la complejidad derivada esencialmente de la globalización, se exige un nivel adecuado no solo de honestidad y responsabilidad en la clase política como antes, sino también una capacidad añadida necesaria en los asuntos públicos (que implica nuevos conocimientos, habilidades y destrezas) según el nivel y cargo ostentado. No es admisible el amauterismo ni el buenismo para ostentar y gestionar determinados puestos y cargos políticos. En este sentido, Natera y Vanaclocha (2015) entienden el proceso de liderazgo político como uno de los elementos de representatividad elemental de la agregación colectiva, y de regulación de conflictos que define a la política. La complejidad actual y la ciudadanía sin duda, demandan a la clase política, además de las virtudes y valores clásicos, una empatía, capacitación, no endogamia, ni sectarismo, y una mayor transparencia. No es admisible improvisar o experimentar sin conocimiento, por ello deben aplicarse unas mínimas cautelas en el ámbito público, ya que sino esto sería una grave e inicial irresponsabilidad.

Así pues, la decisión implica reflexión y análisis como se ha dicho, se trata de un debate interno y plural, de contenido amplio en las instancias decisorias, y previo en sus órganos de consulta y apoyo. En todo caso, siempre existe el peligro de un error en la adopción de decisiones, ahora bien no se equivoca quien no adopta ninguna decisión. Los márgenes posibles y previsibles de errores deben proveerse, así como los medios adecuados previstos para la rectificación (Ramió, 2012). No puede ni debe olvidarse tampoco en última instancia, que toda decisión siempre es prospectiva. 
La adopción de decisiones a su vez no tiene un solo contenido de carácter político, económico o jurídico, sino que también supone otros aspectos, entre los que destacaríamos como más relevantes los siguientes: a) la selección de tareas y objetivos; b) la clarificación y priorización de políticas, tareas y objetivos; c) la selección de indicadores de medición, valoración y monitoreo; d) la asignación y priorización de recursos o efectivos a emplear; e) la fijación de plazos de actuación y del cronograma posible y adecuado general de actuación; f) la priorización de políticas, programas, presupuestos parciales o sectoriales y proyectos públicos; g) la aprobación de un plan posible de calidad en la actuación pública, y en especial en la provisión y prestación de los bienes y servicios públicos; $h$ ) las fórmulas posibles de gestión de los servicios públicos; h) el diseño de un plan de autoevaluación posterior a la implementación; i) el diseño de un plan de dirección y gerencia; y por último, j) un plan de selección, formación, motivación, evaluación y retribución del personal.

Todos estos aspectos mencionados, y otros más de posible interés añadibles, deberían plasmarse, clarificarse y concretarse en un plan de actuación de carácter estratégico de las diversas instituciones públicas. Si la no actuación o parálisis administrativa es grave e irresponsable, y con costes posibles, la decisión se hace necesaria considerando todo lo anterior, aunque no siempre exista la certeza total y absoluta. No siempre tendremos un conocimiento completo de todos los datos y variables a considerar, ya que como ha señalado la doctrina, entre otros Fontaine (2015), sabemos que hay un sin número de variables que no controlamos o que no controlan las personas que toman decisiones. Donde el análisis de políticas públicas puede ser útil, precisamente al minimizar el grado de incertidumbre en el proceso mismo. No obstante, a pesar de un mayor o menor grado de información y de incertidumbre, es necesario adoptar una decisión con todos los riesgos que ello implica; ahora bien, también es posible que la división sea no tomar ninguna decisión y no afrontar el problema con lo que esto supone de riesgos y costes de diverso tipo.

Además de lo anterior, es necesario recalcar que el proceso de adopción de decisiones se enmarca en un marco de referencia mucho más amplio, con la ayuda de la ciencia política, como es sabido, la disciplina que estudia y analiza este hecho. Abundando en lo anterior, entre otros, Casar y Maldonado (2008), han señalado que: «dado que el estudio de la política pública va mas allá que del análisis empírico de ciertas decisiones -de estudios o de casos- y abarca también el análisis de la formación de agendas, de la discriminación de alternativas, de la viabilidad de las mismas y del impacto de las políticas adoptadas (...)» y que «(...) la investigación sobre y el desarrollo de la teoría de la toma de decisiones requiere de la investigación del conocimiento de la ciencia política sobre las instituciones y el comportamiento político (...)» (Casar y Maldonado, 2008: 209). 
Una de las características, y a la vez problemática, de los sistemas políticos contemporáneos es la pérdida de soberanía y de capacidad de decisión de los estados, en virtud del poder y el protagonismo decisorio de los expertos y de los organismos internacionales (Colomer, 2015; Habermas, 2016) resultante de los procesos de globalización y de los avances que la tecnología, la comunicación e información que han sufrido estas últimas décadas. Por esta razón, estamos en presencia de una nueva y diferente tecnocracia, surgida en el primer tercio del pasado siglo XX, denominada "organización científica del trabajo», dando origen al pensamiento y praxis del taylorismo y del fayolismo. Ambos enfoques compuestos fundamentalmente, por ingenieros y expertos en organización, enfrentan los conceptos de democracia y tecnocracia, a la razón del pensamiento dando origen al llamado «fin de las ideologías». Esto supuso de facto entre otros fenómenos, la crítica directa a la incipiente democracia liberal representativa, el cuestionamiento de la necesidad de los partidos políticos como instrumentos válidos y legítimos de la democracia, el ataque al asociacionismo y al sindicalismo, la defensa de la inutilidad de las elecciones y del voto, la defensa del populismo fascista y comunista, y de la inutilidad del debate político por la ciudadanía, considerada menor de edad.

En la actualidad se plantea por causas diferentes el fenómeno de la tecnocracia debido a la crisis de representatividad, la falta de confianza en los políticos, y la carencia de capacitación y liderazgo político entre otros, provocando que durante las últimas décadas el concepto de tecnocracia ha evolucionado tomando nuevos matices como los siguientes: a) la tecnocracia piensa y actúa no solo a nivel nacional, sino a nivel mundial; y b) los representantes del pasado lo eran básicamente, de profesiones vinculadas a la ingeniería industrial, al análisis de organizaciones y de la producción. En cambio, actualmente estos proceden con mayor frecuencia a los ámbitos de la economía, de las finanzas o de las tecnologías de la información y de la comunicación.

La tecnocracia clásica fue definida, entre otros, por Meynaud (1964): «como la remoción del político por el técnico, y más concretamente cuando de una u otra manera el técnico consigue asegurarse la última palabra, y cuando ha logrado la facultad de ser resorte último (...)» (Meynaud, 1964: 257-270) (García Pelayo,1974). El conocimiento clásico proveniente de la filosofía y las humanidades fueron relegadas y despreciadas por los tecnócratas, que también incluyen a las ciencias sociales en ese olvido y menosprecio. La tecnocracia minusvalora el pensamiento racional, y prima solo lo cuantitativo y lo técnico, olvidando los aspectos y valores de las personas en si mismas, así como las dimensiones y comportamientos éticos y sociales contribuyendo en la construcción de una sociedad insolidaria, exclusiva, individualista, economicista y anómica (Habermas, 2016). Los valores propugnados por esta nueva 
tecnocracia son pues la eficiencia, el lucro desmedido y el mercado sin limitaciones; y todo ello, por encima de la sociedad, las personas y la democracia.

Además, subyuga el pensamiento crítico, la investigación y a las universidades al valor supremo y único del mercado, minusvalorando graves problemas mundiales como el desarrollo sostenible, la degradación medioambiental y climática, el desempleo, los fenómenos migratorios o la exclusión social.

De lo precedente hay que significar también, que la visión y la misión de la tecnocracia actual es limitada, ya que es cortoplacista y no estratégica. Por tanto, con un contenido limitado unidimemsional fruto del economicismo que la caracteriza y que a la vez la incapacita de entrada para intentar dar respuestas adecuadas a una sociedad tan compleja y diversa (con unas redes plurales interpersonales y sociales, con gran diversidad de ideas, bases de datos de conocimientos, de demandas y problemas pendientes) como la actual; y ello frente al debate, la razón y la deliberación propias de la democracia participativa.

El neoliberalismo económico y sus valores, reforzado por la globalización, y la digitalización, se opone al liberalismo político democrático y al republicanismo cívico, inspirados por la razón y la Ilustración, y entre cuyas principales manifestaciones está la defensa y la garantía de todos los derechos humanos (no solo los clásicos e iniciales, sino también los actuales llamados de la «cuarta generación»).

En concordancia con lo anterior, señalaríamos lo siguiente propuesto por García Pelayo (1974), que entiende por tecnocracia una estructura de poder en la cual los técnicos condicionan o determinan la toma de decisiones, tendiendo así a sustituir progresivamente al político en la fijaciones las policies, y a los burócratas tradicionales en la operacionalización de las decisiones o en sus decisiones o en su participación en la misma decisión misma. La tecnocracia significa así, la presencia de una «nueva clase política» compuesta por «tecnócratas» que comprende no solo a los técnicos del proceso productivo sino también a los especialistas en management, planificación, organización, investigaciones operacionales, análisis de sistemas, etc.; en una palabra, los entendidos en teoría y prácticas de sistemas. La tecnocracia carece, en términos generales, de institucionalización formal, y su posición en la estructura de poder deriva del hecho de quien es capaz de cumplir una función necesaria para que un sistema dado adquiera los poderes inherentes a tal cumplimiento, con independencia de que ello tenga o no tenga reconocimiento formal (García Pelayo, 1974)

Ahora bien, la influencia y la predeterminación a nivel internacional de las decisiones gubernamentales que se imponen a los gobiernos, no son sólo en materia económico-financiera o comercial, sino en todos los ámbitos de las políticas públicas, como la educación (Fernández Liria, García y Galindo, 2017), la salud, la ecología, etc. En este sentido, entre otros, Colomer (2015) ha señalado que: «los sistemas 
políticos basados en gobiernos estatales y partidistas están perdiendo capacidad de tomar decisiones en políticas públicas, en muchos países, generando amplias coaliciones multipartidistas y expertos técnicos no partidistas ejecutan las directrices de las organizaciones internacionales» (Colomer, 2015: 135) disminuyendo el poder de los estados por crear y planificar agendas gubernamentales adecuadas a los problemas y situación propias de cada uno de los países.

Esta epistemología neoliberal influye en el proceso decisional a nivel mundial en los asuntos públicos priorizando al mercado y no a los estados. Esto no sólo produce la ausencia de una capacidad decisoria en los gobiernos mermando su capacidad directora, sino que disminuye el de la propia soberanía nacional. En el caso de la Unión Europea, una de las críticas y disfunciones más notables de sus intelectuales es la llamada comitología o comitocracia, especialmente en la Comisión, ya que las múltiples reuniones así como los numerosos grupos de expertos contribuyen a la falta de rapidez aportando complejidad pero también a la falta de transparencia, a la influencia y a la patrimonialización de las decisiones de los grupos de interés.

No obstante, otro problema a tener en cuenta en la «regeneración democrática» o en la "democracia de calidad», son las llamadas puertas giratorias y el papel que realizan los grupos de interés ${ }^{1}$ (Berger, 1981; Von Beyme, 1986; Olson, 1991; Molins, Muñoz Márquez y Medina, 2016) actuantes no solo antes de la adopción de decisiones, sino también después en su ejecución. El papel de influencia de los grupos de presión será inversamente proporcional a la existencia, o no, de la fortaleza institucional pública y de su regulación y control adecuada.

\section{La importancia del papel del llamado «centro de gobierno» en el proceso decisional de los centros de poder}

En los últimos años, se ha enfatizado mucho en destacar la importancia de una nueva variable a considerar en el análisis del proceso decisional, siendo lo que la doctrina ha denominado «centros de gobierno» o «el gobierno del gobierno» (Aguilar Villanueva, 2013). El auténtico núcleo duro de este centro de poder. Este término no debe confundirse por el empleado por el poder ejecutivo en referencia a los gabinetes políticos, de los grupos parlamentarios o de los partidos políticos y de sus fundaciones, aunque exista entre todos estos una posible relación y colaboración.

El término gobierno, en un sentido moderno y más amplio que el clásico jurídico, implica un conjunto integrado de estructuras y órganos administrativos en cuya composición, funciones y comportamiento, intervienen un conjunto diverso de 
reglamentaciones, protocolos, usos y costumbres, de carácter formal y, o informal. Este complejo orgánico muchas veces, no es suficientemente transparente y abierto siendo a veces, solo simbólico y formal.

No debe confundirse el gabinete del Primer Ministro o Presidente del Ejecutivo, con éste ya que según Alessandro et al. (2014): «el Centro de Gobierno, en un sentido estricto se refiere, a las organizaciones y unidades que proporcionan un apoyo directo al Jefe del Ejecutivo del país (Presidente o Primer Ministro) generalmente para la gestión política de las actuaciones del gobierno, la gestión estratégica de sus prioridades, la coordinación del diseño de las políticas, la conducción de su implementación, el monitoreo del desempeño y la gestión del cumplimiento y la comunicación de resultados» (Alessandro et al., 2014:16).; además añade que «el centro de gobierno es el timón del gobierno, capaz de impulsar sus objetivos prioritarios de manera coherente. Puede ser un valioso mecanismo de transmisión para asegurar que las políticas prioritarias del gobierno sean efectivamente implementadas y produzcan resultados para los ciudadanos» (Alessandro et al., 2014:16).

Por su parte, la OCDE (2015) ha indicado al respecto que «el gobierno abierto incide en una gran diversidad de campos y abarca una amplia gama de principios y de prácticas cuyas finalidades son hacer que la relación entre los gobiernos y los ciudadanos sea más dinámica, mutuamente benéfica y basada en una confianza recíproca. Las iniciativas del gobierno abierto pueden ayudar a mejorar la transparencia, impulsar la innovación en el sector público, empoderar a las personas y volver los procesos de las políticas públicas más representativos, incluyentes y efectivos» (OCDE, 2015:3).

El anterior citado autor y sus colaboradores caracterizan el centro de gobierno desde una perspectiva amplia y general del gobierno, que no tiene ningún ministerio ni agencia sectorial. Además de destacar que, éste tiene el empoderamiento político otorgado por su estrecha conexión con el jefe del Ejecutivo (Alessandro et al., 2014).

Vemos pues que esta nueva variable a considerar, además de novedosa, hace más complejo el proceso decisional público en general, y en especial en lo relativo a creación de las agendas de las políticas públicas. Este factor añadido pone de manifiesto que ésta aporta una visión global, integral u holística de la gobernanza actual.

Ahora ese centro de gobierno, no puede ser un poder dentro del poder, no transparente, sino que no puede ni debe olvidar su papel y carácter de apoyo y asesoramiento al gobierno legítimo strictu sensu y a su presidente, que ostentan la legitimidad y representación democráticas, al servicio de y por la ciudadanía.

Además, al centro de gobierno le corresponderá también, ejercer las tareas de liderazgo institucional de coordinación y de dirección vertical y horizontal de todos los órganos y unidades, políticas y programas del gobierno. 
El liderazgo político necesario, no solo será fruto de la actual personificación de la política, que implica entre sus habilidades y destrezas, las de; comunicación, relación, integración, dirección de equipos, y empatía con la ciudadanía, etc. La elección legítima y democrática inicial no es suficiente, sino que el electo debe ejercitar además el liderazgo, para desarrollar el jefe del ejecutivo, la legitimación añadida de su ejercicio democrático pleno. Sobre la figura actual de los presidentes del gobierno, puede verse entre otros: que la fase o etapa del proceso de decisiones sea clara y precisa, cara a su comprensión y ejecución, y que las fases posteriores de su formulación y comunicación también lo deben ser.

Además, cómo poder evaluar ex post de forma completa y rigurosa la acción pública y las políticas públicas, y por ende en su caso exigir responsabilidades fruto de la rendición de cuentas, si su diseño derivado de la decisión no es clara y bien conocida.

\section{Conclusiones}

En las páginas precedentes hemos hecho una aproximación sintética y reflexiva, acerca de la fase del proceso de decisiones en la acción pública en general, y de las políticas públicas en particular; centrándonos básicamente en el poder ejecutivo y no en otros actores o instituciones político-administrativas. No obstante, un gran número de los aspectos aquí tratados son aplicables por analogía al poder legislativo $\mathrm{y}$ al poder judicial, los cuales tienen sin duda su propia idiosincrasia.

Es indudable que el proceso de adopción de decisiones es complejo y con incertidumbres, que se intensifican fundamentalmente como consecuencia de dos fenómenos destacables: a) la aparición de nuevos y diversos actores en la arena política; b) la desaparición del poder decisorio monopolista y protagónico del Estado. El sistema político-administrativo ha pasado, de ser estatocéntrico a ser policéntrico, desde una visión centralizada a una opción plural y descentralizada, basada en la participación de la ciudadanía en los procesos de toma de decisión y agenda de las políticas públicas.

El proceso de adopción de decisiones nunca fue fácil, ya que implica conocimientos, habilidades y destrezas clásicas, acordes con la realidad y dinámica social actual, que exige no solo seriedad y rigor, sino también una capacitación adecuada de los decisores y asesores. Adoptar decisiones es en parte una síntesis entre conocimientos y experiencia, y en tiempos de crisis además, la incertidumbre y la ansiedad son obviamente mayores, lo que complejiza el proceso decisional.

Por otro lado, como ya también se ha señalado, el proceso de toma de decisiones y su resultado deben ser rigurosos, claros sin ambigüedades en su diseño y comunicación. No cabe duda de que las decisiones adecuadas contribuirán de forma notable 
a una mejora de la calidad de la democracia pudiendo decir, que este es un medio para un fin.

Finalmente y para concluir, queremos citar y hacer nuestras las siguientes reflexiones consideradas como esenciales, siendo: a) «Lo público presenta por su propia naturaleza, distintos problemas para definirse y funcionar. Esto no siempre es sencillo porque la decisión pública está siempre sometida a tensiones que provienen de la esfera política, de la propia administración, del mercado y de los distintos grupos de interés que operan en torno a los problemas públicos. A esto hay que sumarle que, en ocasiones el interés de la sociedad no coincide con la voluntad expresada de ésta» (Bañón y Jiménez, 2008:13); b) «(...) en la actualidad ya no se puede hablar de una autonomía decisoria de los poderes públicos, sino que, para que la acción sea efectiva, ha de contar con el apoyo de los distintos grupos sociales. La cooperación de los poderes públicos con su entorno para mantener el equilibrio social, lo que se denomina gobernanza, se extiende desde la toma de decisiones hasta el desarrollo de la acción pública» (Bañón y Jiménez, 2008: 13); c) «las políticas públicas y las decisiones que conllevan, están dentro del campo del poder que es el Estado, lleno de conflictos internos e influencias externas de todo tipo, que son causas de las decisiones públicas» (Fontaine, 2015: 31); y d) en un régimen democrático, el gobierno actúa en nombre del interés general. Sus políticas son el reflejo de las preferencias partidarias e ideológicas que se manifiestan en la política, en el día a día y aquellos momentos especiales que constituyen las elecciones. Las decisiones que atañen a una política pública son tomadas al término de un debate de ideas razonables y racional» (Fontaine, 2015:33).

\section{Referencias bibliográficas}

AGUILAR VILLANUEVA, L. F. (2013) El gobierno del Gobierno. México: Fondo de Cultura Económica. http://www.inap.mx/portal/images/pdf/book/gobierno_ delgobierno.pdf

ALESSANDRO, M.; M. LAFUENTE y C. SANTISO (2014) Gobernar para cumplir con los ciudadanos. El rol del Centro de Gobierno en América Latina y el Caribe. Washington: BID.

BAENA DEL ALCÁZAR, M. (2004) Curso de Ciencia de la Administración. Madrid: Tecnos.

BAUMAN, Z. (2004) Modernidad líquida. Buenos Aires: FCE.

BAÑÓN, R. y S. JIMÉNEZ (2008) La Nueva Gestión Publica. Madrid: TQM.

BERGER, S. (1981) Organizing Interest in Western Europe. Cambridge: Cambridge University. 
CANALES ALIENDE, J. M. (2015) Estudios sobre la democracia, el gobierno y la administración pública. Madrid: Editorial Universitas y Observatorio Lucentino de Administración y Políticas Públicas Comparadas (OLAPPC) de la Universidad de Alicante.

CANALES ALIENDE, J. M. y A. ROMERO TARÍN (2014) «El liderazgo político», en J. M. CANALES ALIENDE y J. J. SANMARTÍN PARDO (Ed.), Introducción a la ciencia política, Madrid: Universitas, 243-251.

CASAR, M. A. y C. MALDONADO (2008) Formación de la agenda y procesos de toma de decisiones: una aproximación desde la ciencia política. México: CIDE, núm. 207

COLOMER, J. M. (2015) El gobierno mundial de los expertos. Barcelona: Anagrama.

CURTIS, S. (2018) «Las ciudades globales y el futuro del orden mundial» en el dossier núm. 67, de enero-marzo: El poder de las ciudades. Barcelona: La Vanguardia.

FERNANDEZ LIRIA, C.; O. GARCIA y E. GALINDO (2017) Escuela o barbarie. Entre el neoliberalismo salvaje y el delirio de la izquierda. Madrid: Akal.

FONTAINE, G. (2015) El análisis de políticas públicas. Barcelona: Anthropos y FLACSO Ecuador. https://doi.org/10.18601/16578651.n18.12

GARCÍA PELAYO, M. (1974) Burocracia y Tecnocracia. Madrid: Alianza Editorial.

GÓMEZ CALVO, V. y A. ROMERO TARÍN (2016) Hacia una nueva gobernanza: los poderes públicos y el Tercer sector. Una relación necesaria pero inexistente. Barataria. Revista Castellano-Manchega de Ciencias Sociales, núm. 21, 163-173. https://doi.org/10.20932/barataria.v0i21.307

GONZÁLEZ, F. (2013) En busca de respuestas. El liderazgo en tiempo de crisis. Barcelona: Debate.

HABERMAS, J. (2016) En la espiral de la tecnocracia. Madrid: Trotta.

KINGDON, J. W. (1984) Agendas, Alternatives and Public Policy. Boston: Little Brown.

LINDBLOM, Ch. E. (1994) El proceso de elaboración de politicas públicas. Madrid: INAP.

MARTÍNEZ PUÓN, R. (2011). Directivos versus Políticos. La importancia de la función directiva en las Administraciones Publicas. México: Editorial Miguel Ángel Porrúa.

MÉNDEZ, J. L. (2013) Liderazgo Político. México: Siglo XXI.

MÉNDEZ JUEZ, M. (2016). La Economía Digital de los Datos Públicos en Democracia: Una visión institucional. Pamplona: Thomson Reuters.

MEYNAUD, J. (1964). La Tecnocracia. Madrid: Tecnos.

MOLINS, J.; L. MUÑOZ MÁRQUEZ y I. MEDINA (2016) Los Grupos de Interés en España. La influencia de los lobbies en la política española. Madrid: Tecnos. 
NATERA, A. y F. J. VANACLOCHA (2005) Los liderazgos políticos en el mundo político y la gestión pública. Madrid: Universidad Carlos III de Madrid y BOE.

OCDE (2001) Citizens as partners. Handbook on information, consultation and Public Participation in policy-making. Governance. Paris: OCDE https://doi. org/10.1787/9789264195578-en

OCDE (2015) Gobierno Abierto en América Latina. Paris: OCDE. https://doi. org/10.1787/9789264225787-4-es

OLSON, M. (1992) La lógica de la acción colectiva: Bienes públicos y teoría de los grupos. México: Limusa (Primera edición 1965).

OSTROM, E. (2011) El gobierno de los comunes. La evolución de las instituciones de acción colectiva. 2 sd. Edición. México: FCE, UNAM, IIS.

PASQUINO, G. (2000) La democracia exigente. Madrid: Alianza Editorial.

PETERS, B. G. (2005) «Gobernanza y burocracia pública: ¿nuevas formas de democracia o nuevas formas de control?» Foro internacional, vol. XIV, núm. 4, octubre-diciembre, 585-598.

PETERS, G. y P. PIERRE. (2002) «La gobernanza en niveles múltiples: ¿un pacto fáustico?» Foro Internacional, julio-septiembre. México: Colmex, 429-453.

PONT VIDAL, J. (2017) La innovación en la gestión pública. Madrid: Catarata.

RAMIÓ, C. (2012) La extraña pareja. La procelosa relación entre políticos y funcionarios. Barcelona: Los Libros de la Catarata.

ROMERO TARÍN, A. y G. FONTAINE (2017) «Globalización, gobernanza y diseño de políticas públicas», en J. M. CANALES ALIENDE, G. FONTAINE y A. ROMERO TARÍN (Eds.). La transformación de los sistemas políticos y de los Estados. La visión y retos en Europa y América Latina en el contexto de la globalización. Barcelona: Anthropos. 59-73.

VON BEYME, K. (1986) Los grupos de interés en la democracia. Buenos Aires: Belgramo.

ZITTOUN, P. (2009) «Understanding Policy Change as a Discursive Problem». Journal of Comparative Policy Analysis, núm.11, 65-82. https://doi. org/10.1080/13876980802648235 